\title{
Neurobiologie der Emotionsregulation
}

Fragestellung: Was sind die neurobiologischen Mechanismen, die nicht suizidalem selbstverletzenden Verhalten bei Patienten mit Borderline-Persönlichkeitsstörung (BPS) zugrunde liegen?

Hintergrund: Selbstverletzungen ohne Suizidabsicht sind ein psychopathologisches Merkmal bei der BPS. Ein häufiges Motiv ist die Reduktion von Anspannung und hohem Stress.

Patienten und Methodik: 21 Frauen mit BPS und 17 gesunde Kontrollpersonen wurden einem psychologischen Stressor ausgesetzt. Danach erfolgte entweder ein kleiner Einschnitt am Unterarm oder eine Scheinintervention. Anschließend wurde eine funktionelle MRT in Ruhe durchgeführt und parallel die subjektive Anspannung und die Herzfrequenz gemessen.

Ergebnisse: Die Inzision beeinflusste signifikant subjektiven und objektiven Stress. BPS-Patientinnen zeigten eine größere Anspannungsreduktion nach Inzision versus Scheininzision. Als biologisches Korrelat zeigte sich bei BPS eine größere Reduktion der Amygdalaaktivität und

Reitz S, Kluetsch R, Niedtfeld I et al. Incision and stress regulation in borderline personality disorder: neurobiological mechanisms of self-injurious behaviour. Br J Psychiatry 2015; 207: 165-72 eine Normalisierung der funktionellen Verbindung zwischen Amygdala und superiorem frontalen Gyrus.

Schlussfolgerungen: Schnittverletzungen vermindern das

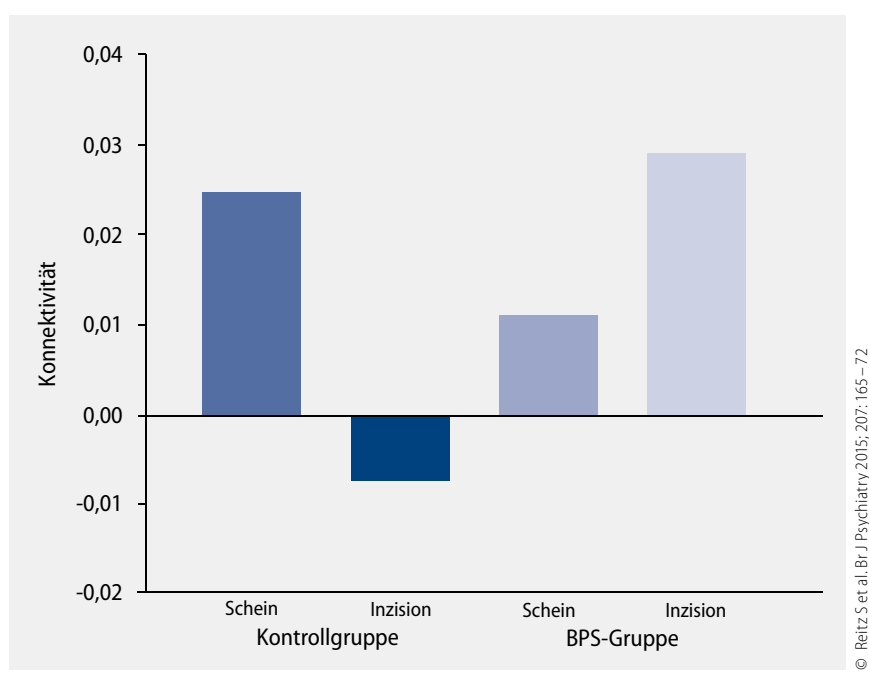

1 Funktionelle Konnektivität zwischen Amygdala und superiorem frontalen Gyrus (BA8) normalisiert sich nach Schnittverletzung in der BPS-Gruppe. Das gegenteilige Bild zeigt sich in der gesunden Kontrollgruppe.

Stressniveau und verringern die Amygdalaaktivität bei Patientinnen mit BPS. Dieser biologische Nachweis der emotionsregulierenden Wirkung von Selbstverletzungen hilft, diese Verhaltensweise bei Patientinnen mit BPS besser zu verstehen.

\section{- Kommentar von Stefan Röpke, Berlin}

\section{Selbstverletzungen bei Borderline-Patienten besser verstehen}

Die anspannungsregulierende Wirkung von Schnittverletzungen bei Patienten mit Borderline-Persönlichkeitsstörung (BPS) ist dem Kliniker gut bekannt. Aufbauend auf einer Reihe von wissenschaftlichen Arbeiten zur emotionsregulierenden Wirkung von Schmerzreizen bei Patienten mit BPS untersucht diese Studie weitere zugrunde liegende neurobiologische Mechanismen. Mit einer sehr innovativen, ökologisch validen Methodik, abgeleitet aus der Blutungszeitbestimmung mittels Inzision, konnte erstmals die direkte Wirkung von Gewebeläsion mittels Schnittverletzung auf Stress und Anspannung im funktionellen Kernspinn bei Patientinnen mit BPS untersucht werden. Als zusätzlicher neurobiologischer Parameter wurde die Herzfrequenz gemessen. Eine erhöhte Amygdalaaktivität, die bei BPS-Patienten vorbeschrieben ist, wurde besonders stark durch die Inzision reduziert, ein Befund, der diese Verhaltensweisen mit erklären kann. Zusätzlich konnte ein vermehrte funktionelle Kopplung (wenn auch nur auf Trendniveau) zwischen emotionsverarbeitenden Hirnregionen (Amygdala) und Regionen, die der kognitiven Kontrolle zugesprochen werden (superiorer frontaler Gyrus), gefunden werden. Ein Hinweis, dass unter Stressbedingungen die kognitive
Kontrolle bei BPS-Patienten eingeschränkt ist und durch Schnittverletzung wieder normalisiert wird. Einschränkend, wie von den Autoren vermerkt, ist das Design durch die einmalige Schnitt- beziehungsweise Scheinschnitt-Bedingung. Aus diesem Grund konnte nur der Zeitverlauf nach Inzision untersucht werden. Nach diesen wichtigen Befunden wäre es interessant die stressregulierende Wirkung eingeübter funktionaler Stressregulationsfertigkeiten, wie sie zum Beispiel im Rahmen der Dialektisch Behavioralen Therapie (DBT) vermittelt werden, neurobiologisch im Vergleich zu untersuchen.

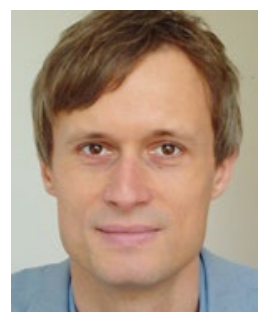

PD Dr. med. Stefan Röpke, Berlin

Oberarzt, Bereichsleiter Persönlichkeitsstörungen und Posttraumatische Belastungsstörung sowie Autismusambulanz, Klinik für Psychiatrie und Psychotherapie, Charité Universitätsmedizin Berlin, Campus Benjamin Franklin. E-Mail: stefan.roepke@charite.de 